\title{
Advances in Cement Research: Referees 2019
}

The following is a list of referees who have reviewed papers for Advances in Cement Research between 30 November 2018 and 15 November 2019. The Institution of Civil Engineers is very grateful for their assistance.

We are continually looking for suitable reviewers for papers submitted to Advances in Cement Research. Papers published in Advances in Cement Research must be submitted to at least two independent referees to judge accuracy, style, impact, importance and interest.

If you are interested in reviewing articles on any topic related to Advances in Cement Research, please submit your name, qualifications or

Hakim Salem Abdelgader HA Abdel-Gawwad

Mohd Mustafa Al Bakri Abdullah

Ahmed Adawy

Sam Adu-Amankwah

Satish Agarwal

Mohamed Amin

Costas Anagnostopoulos

Özge Andiç-Çakır

Carmen Andrade

Mathieu Antoni

Denise Antunes da Silva

Ioan Ardelean

Chris Atkins

Serdar Aydın

A Bahurudeen

Magdalena Balonis

Phil Banfill

K Baskaran

Mohsen Ben Haha

Arnon Bentur

Susan Bernal López

Leon Black

Jeffrey Bullard

Oswaldo Burciaga-Diaz

Juliana Calabria-Holley

Mingli Cao

Fabio Cardoso

Serhat Celikten

Piyush Chaunsali

Bing Chen

Xiao Chen
Ramesh Chouhan
Deborah Chung
Ping Cui
Tobias Danner
Thomas Dyer
Dominique Ectors
Adel el Turki
MS El-Feky
Tian Zi Eng
Kendra Erk
Claudio Ferone
Krassimir Garbev
Emilio Garcia-Taengua
Daniel Geddes
Fred Glasser
Seishi Goto
Theodore Hanein
Edris M Hassan
Chuanlin Hu
Zhangli Hu
Ying Hu
Edgardo Irassar
Sivakumar Kandasami
Xinyuan Ke
Mehmet Kirgiz
Mohammed Haneefa Kolakkadan
Konstantinos Kolovos
Ramaswamy Koodalloor
Parasuraman
João Labrincha

Gwenn Le Saout

Lei Lei

Qing-feng Liu

Yuanyuan Liu

Barbara Lothenbach

Duyou Lu

Bin Ma

Ricardo Magallanes-Rivera

Sagrario Martinez-Ramirez

Marcelo Medeiros

Sean Monkman

M Murugan

Ebrahim Najafi Kani

Meysam Najimi

Moncef Nehdi

Kamal Neupane

Milena Pavlíková

Johann Plank

John Provis

Ramadhansyah Putra Jaya

hamid rahmani

Alaa Rashad

Deepak Ravikumar

Sylvie Rossignol

Francisco José Rubio-Hernández

Md. Safiuddin

Mustafa Sahmaran

Aref Samadi-Dooki

Mercedes Sanchez

Manu Santhanam

Ashok Santra
Prabir Sarker

Geetha Selva

Nasir Shafiq

Zhenguo Shi

Tao Shi

Myongshin Song

Tongbo Sui

Prannoy Suranen

Elena Tajuelo Rodriguez

Lei Tao

Antonio Telesca

Brett Tempest

Neven Ukrainczyk

Kunamineni Vijay

Baomin Wang

Mark Whittaker

Frank Winnefeld

Bi Yun Zhen Wu

Kazuo Yamada

Peiyu Yan

Bin Yuan

Maciej Zajac

Qiang Zeng

Jun Zhang

Wensheng Zhang

Chunsheng Zhou

shuguang zhou

Emilio Zornoza 